\title{
PANTHERE V2: MULTIPURPOSE SIMULATION SOFTWARE FOR 3D DOSE RATE CALCULATIONS
}

\author{
Gaël PENESSOT ${ }^{1 a}$, Éléonore BAVOIL ${ }^{1}$, Laurent WERTZ ${ }^{1}$, Fadhel MALOUCH ${ }^{2}$, Thierry VISONNEAU ${ }^{2}$ and Julien DUBOST ${ }^{3}$ \\ ${ }^{1}$ EDF/SEPTEN/TE/RP, 12-14 avenue Antoine Dutriévoz, 69628 Villeurbanne, France \\ ${ }^{2}$ CEA-Saclay DANS/DM2S/SERMA, 91191 Gif-sur-Yvette, France \\ ${ }^{3} \mathrm{CS}-\mathrm{SI}, 45$ rue Sainte Geneviève, 69006 Lyon, France
}

\begin{abstract}
PANTHERE is a multipurpose radiation protection software developed by EDF to calculate gamma dose rates in complex 3D environments. PANTHERE takes a key role in the EDF ALARA process, enabling to predict dose rates and to organize and optimize operations in high radiation environments. PANTHERE is also used for nuclear waste characterization, transport of nuclear materials, etc. It is used in most of the EDF engineering units and their design service providers and industrial partners.
\end{abstract}

\section{Introduction}

PANTHERE is a radiation protection code used for 3D simulation of gamma dose rates and fluence for complex environment with distributed radioactive sources like nuclear facilities.

PANTHERE is intensively used by EDF engineering units and its industrial partners. It was originally used to prepare maintenance and modification operations in nuclear power plants, especially on the primary circuit like pressurizer heaters replacement and steam generator replacement. It is now extended to other fields like:

- shield design and zoning for EPR reactors

- dose calculations for the qualification of PWR equipments during severe accidents.

- decontamination and dismantling operations

- radiological characterization for applications associated to transport and nuclear waste management

PANTHERE is verified and validated by comparison with the reference Monte Carlo code TRIPOLI- 4 of the CEA and gamma dose rates experimental measurements.

\section{Physical models used}

PANTHERE's computation kernel uses CEA solver NARMER, which is linked to the EDF R\&D geometric library LIBCAD. This chapter focuses on the different physical models used by NARMER.

\subsection{The point kernel integration method}

The method used in NARMER is the point-kernel integration method with build-up factors. It is a macroscopic approach for gamma dose rate calculation,

\footnotetext{
a Corresponding author: gael.penessot@edf.fr
}

taking into account the interaction with matter. The dose equivalent rate (DER) for a source of activity A (gamma.cm $\mathrm{cm}^{-3} \cdot \mathrm{s}^{-1}$ ) in a volume $\mathrm{V}_{\mathrm{s}}$ is computed as in Eq. (1):

$$
D E R(E)=\sum_{0}^{E_{\max }} F_{D}(E) \int_{0}^{V}\left[B(E) \frac{A(E) e^{-\sum_{i} \mu_{i}(E) x_{i}}}{4 \pi\left(\sum_{i} x_{i}\right)^{2}}\right] d V
$$

with:

- $F_{D}(E)$ the gamma fluence-to-dose conversion factor

$-\mu(E)$ the linear attenuation coefficient

- $x$ the shield thickness encountered by a gamma ray along a straight line

- $d$ the distance between the source and the calculation point

$-B(E)$ the build-up factor

\subsection{The build-up factors}

The build-up factors (B) are used to estimate the scattered radiation in matter. These factors are tabulated for all simple elements (from $Z=1$ to 98) and for 195 energy groups between $15 \mathrm{keV}$ and $10 \mathrm{MeV}$. For multi-layer shields, the build-up factors are computed according to the "CEA/SERMA" formula [1].

\section{PANTHERE architecture}

PANTHERE is constituted of three main components: a computation kernel, a MySQL database for models management and sharing, and a 3D GUI to define the studies and both geometrical and radiological environment. It runs on a Linux operating system. 


\subsection{Pre-treatment in the GUI}

PANTHERE GUI allows the definition of geometrical and radiological models by the use of 9 parametrical primitives (e.g., cylinder, wall, etc.), frequently used to model nuclear facilities. They can be composed of 1 to 5 distinct media: 2 internal media (air/water) and 3 successive layers: "metal", "thermal insulation" and "biological protection".

Radioactive sources can be added to every layer of each object (both volume and surface sources). The spectrum is defined with energy rays, with nuclides or using energy groups. The nuclear data are taken from JEF2.2 [2].

Observation points are defined by their 3D coordinates. Measurement results can be added to each observation point to perform a readjustment of the predefined source activity (fitting).

Finally, results can be given according to several response functions: the equivalent dose rate $\mathrm{H}^{*}(10)$ in $\mathrm{mSv} \cdot \mathrm{h}^{-1}$, the "kerma in air" $\mathrm{mGy} \cdot \mathrm{h}^{-1}$, the energetic fluence rate $\mathrm{MeV} \cdot \mathrm{cm}^{-2} \cdot \mathrm{s}^{-1}$ and the uncollided flux gamma.cm ${ }^{-2} \cdot \mathrm{s}^{-1}$.

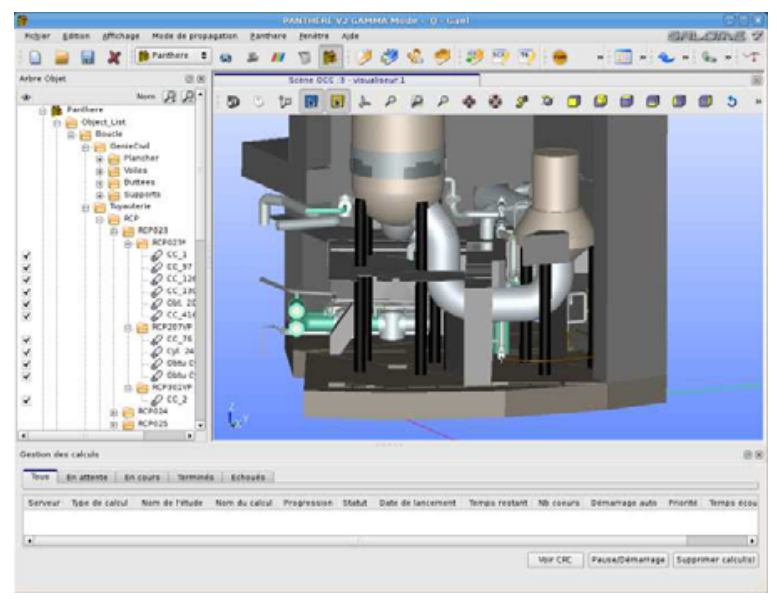

Figure 1. Caption of the PANTHERE v2 GUI

\subsection{Post-processing in the GUI}

The results of the computation are displayed in form of source/point or nuclide/point matrix and can be exported in csv format or opened in a spreadsheet editor. Dedicated SALOME [3] modules allow for radiological zoning and isodose mapping.

PANTHERE results, combined with NPP maintenance program can be used as an input of CADOR, an optimization code for the installation of protection on NPP contaminated pipes. This code perform an optimization of the collective dosimetry by scheduling the task in order to reduce both painfulness of team in charge of installing protection and the dosimetry of team in charge of maintenance.

\section{Physical model limitations}

PANTHERE uses a simplified physical model to enhance calculation performance (10 to 25 times faster than stochastic methods like TRIPOLI-4). However, it restricts the usage of PANTHERE to domains where the approximations of Eq. (1) are pertinent.

The main restrictions are geometrical: the singularity of Eq. (1) imposes a minimum distance between source and observation point.

Finally, the build-up factors are validated for penetration lengths up to $50 \mathrm{MFP}$, and for energy ranging from $15 \mathrm{keV}$ to $10 \mathrm{MeV}$. They should not be used outside this validation domain.

Fortunately, this domain covers most of studies for nuclear energy facilities.

\section{Functionalities}

\subsection{CAD import}

PANTHERE inherit SALOME's CAD modeler GEOM which allow s designing buildings, structures and piping. Moreover, PANTHERE allows the user to import CAD data from specific modelers like SolidWorks or PDMS. This functionality improves the efficiency of the geometrical modeling.

\subsection{Source readjustment module}

In most cases, calculations are compared to measures. PANTHERE features a module that adjusts theorical activity in model's sources to fit experimental measures. It allows the user to have a more realistic model and more relevant source contributions in order to design shelding.

\subsection{Computational performance}

The kernel of PANTHERE v2 improves computational performance by using an optimized geometrical representation and raytracer, and by implementing shared memory parallelization over the observation points, the sources and the nuclides.

Further acceleration could be obtained by distributed computing currently under development.

\section{Conclusions}

PANTHERE plays an important role in an ALARA regulatory process by providing simulation results for industrial radiation protection. Its use of a simplified computation method and its user-friendly GUI dedicated to radiation protection allow a reliable and fast industrial usage to maintain occupational radiation exposures as low as reasonably achievable in nuclear facilities.

Moreover, future releases will include beta (short term) and neutron (long-term) dose rates. 


\section{References}

[1] C. Suteau, M. Chiron, G. Arnoud, Improvement of MERCURE-6's General Formalism for Calculating Gamma-Ray Build-up Factors in Multilayer Shields, Nucl. Sci. \& Eng. 147 (2004), pp.419-424.

[2] JEF-2.2 Nuclear Data Library, NEA JEFF Report 17 (2000)

[3] http://www.salome-platform.org 